\title{
Indicadores de qualidade da terapia nutricional enteral são ferramentas úteis para o monitoramento em pacientes com câncer avançado em cuidados paliativos?
}

\author{
Are quality indicators of enteral nutritional therapy a useful tool for monitoring patients with ad- \\ vanced cancer in palliative care?
}

DOI: $10.37111 /$ braspenj.2020354012

Kissila Ferreira de Souza

Mariana Fernandes Costa

Rosane de Souza Santos ${ }^{3}$

\begin{abstract}
Unitermos:
Indicadores de Serviços. Indicadores de Qualidade em Assistência à Saúde. Qualidade da Assistência à Saúde. Nutrição Enteral. Terapia Nutricional. Cuidados Paliativos. Neoplasias.
\end{abstract}

\section{Keywords:}

Indicators of Health Services. Quality Indicators, $\mathrm{He}$ alth Care. Quality of Health Care. Enteral Nutrition. Nutrition Therapy. Palliative Care. Neoplasms.

\section{Endereço para correspondência:}

Rosane de Souza Santos

Instituto Nacional de Câncer José Alencar Gomes da Silva - Hospital do Cancer IV, Setor de Nutrição Clínica Rua Visconde de Santa Isabel, 274 - Vila Isabel - Rio de Janeiro, RJ, Brasil - CEP: 20560-121

E-mail: rosanerssoli@gmail.com

\section{Submissão:}

5 de março de 2020

Aceito para publicação:

10 de dezembro de 2020

\begin{abstract}
RESUMO
Introdução: A terapia nutricional enteral (TNE), em pacientes em cuidados paliativos exclusivos, deve estar direcionada a uma melhor qualidade de vida, sendo importante sua monitorização para que o paciente tenha acesso ao melhor que a terapia possa lhe oferecer. $O$ objetivo deste estudo foi aplicar os indicadores de qualidade da TNE em pacientes com câncer avançado em cuidados paliativos. Método: Estudo descritivo, observacional, de abordagem quantitativa. Foram aplicados sete indicadores de qualidade da TNE propostos pelo International Life Sciences Institute do Brasil e quantificados os motivos da suspensão ou interrupção da dieta enteral durante a internação. Resultados: Participaram 51 pacientes, internados no período de maio a novembro de 2019. As frequências de saída inadvertida de sonda de nutrição enteral $(0,02 \%)$, dias de administração adequada do volume prescrito versus volume infundido $(92,6 \%)$ e diarreia $(2 \%)$ estiveram dentro da meta estabelecida. A administração de proteína $(25,5 \%)$ e energia $(57,4 \%)$, a frequência dos episódios de distensão abdominal $(33,3 \%)$ e constipação intestinal $(86,3 \%)$ estiveram inadequados. A dieta foi interrompida ou suspensa principalmente devido à ocorrência de vômitos, cuidados ao fim de vida ou obstrução de sonda. Conclusão: $O$ uso dos indicadores de qualidade da terapia nutricional se mostrou viável nesse grupo de pacientes. Algumas inadequações encontradas nesta população podem estar associadas à mudança de objetivo da terapia nutricional nos pacientes com menor expectativa de vida. Adaptações baseadas no prognóstico podem ser necessárias, além da aplicação de novos indicadores, ou adequações das metas propostas.
\end{abstract}

\section{ABSTRACT}

Introduction: Enteral nutritional therapy (ENT) in patients with advanced cancer in palliative care should be directed towards a better quality of life, being important its monitoring so that the patient has access to the best that the therapy can offer. The objective of this study was to apply the quality indicators of ENT in patients with advanced cancer in palliative care. Methods: Descriptive, observational study with a quantitative approach. Seven ENT quality indicators proposed by the International Life Sciences Institute in Brazil were applied and the reasons for the suspension or interruption of the enteral diet during hospitalization were quantified. Results: 51 patients admitted from May to November 2019 participated in the study. The frequencies of inadvertent exit of enteral nutrition tube $(0.02 \%)$, the days of adequate administration of the prescribed volume versus infused volume $(92.6 \%)$ and diarrhea $(2 \%)$ are within the goal used. The administration of protein $(25.5 \%)$ and energy $(57.4 \%)$, the frequency of episodes of abdominal distension $(33.3 \%)$ and constipation $(86.3 \%)$ were inadequate. The diet was interrupted or suspended mainly due to the occurrence of vomiting, end-of-life care or tube obstruction. Conclusion: The use of quality indicators of nutritional therapy proved to be viable in this group of patients. Some inadequacies found in this population may be associated with the change in the objective of nutritional therapy in patients with shorter life expectancy. Adaptations based on the prognosis may be necessary, in addition to the application of new indicators, or adjustments to the proposed goals.

1. Nutricionista; Especialista em oncologia; Instituto Nacional de Câncer José Alencar Gomes da Silva (INCA), Rio de Janeiro, RJ, Brasil.

2. Doutora em Alimentação, Nutrição e Saúde, Instituto Nacional de Câncer José Alencar Gomes da Silva (INCA), Unidade de Cuidados Paliativos, Rio de Janeiro, RJ, Brasil.

3. Mestre em Neurociências, Instituto Nacional de Câncer José Alencar Gomes da Silva (INCA), Unidade de Cuidados Paliativos, Rio de Janeiro, RJ, Brasil. 


\section{INTRODUÇÃO}

A força tarefa do International Life Sciences Institute do Brasil (ILSI-Brasil) publicou recentemente 32 indicadores de qualidade em terapia nutricional propostas por especialistas em Nutrição Clínica. Esses indicadores são instrumentos utilizados para acompanhar e medir resultados, com o objetivo de melhorar condutas e a assistência ao paciente em muitos centros de saúde, proporcionando uma nutrição adequada e evitando complicações'. Entretanto, poucos estudos publicaram o emprego destes na supervisão e monitoramento da terapia nutricional enteral (TNE) no câncer ${ }^{2,3}$. Além disso, não foram encontrados estudos sobre o seu uso em paciente com câncer avançado em cuidados paliativos exclusivos.

Os cuidados paliativos representam uma ciência recente no Brasil, definida pela Organização Mundial de Saúde como "uma abordagem que melhora a qualidade de vida de pacientes (adultos e crianças) e famílias que enfrentam problemas associados a doenças que ameaçam a vida. Previne e alivia o sofrimento através da identificação precoce, avaliação correta e tratamento da dor e outros problemas físicos, psicossociais ou espirituais"4.

Apesar dos avanços tecnológicos relacionados ao diagnóstico e tratamento do câncer, a doença pode se tornar refratária à terapia antineoplásica. Em países menos desenvolvidos, uma alta parcela dos pacientes iá é diagnosticada em estágio avançado da doença, quando os tratamentos curativos já não apresentam efetividade ${ }^{5}$. Dessa forma, em pacientes com câncer em cuidados paliativos exclusivos, devido à doença avançada, são comuns intercorrências como náuseas, vômitos, xerostomia, disfagia, odinofagia e inapetência, fatores que, muitas vezes, impossibilitam a alimentação oral adequada. Associado a isso, o metabolismo anormal ocasionado pelo câncer favorece um balanço energético e proteico negativo, contribuindo para uma depleção do estado nutricional, levando a um comprometimento funcional progressivo e pior qualidade de vida ${ }^{6,7}$. Todavia, a indicação e manutenção da nutrição enteral via sondas ou ostomias em pacientes em cuidados paliativos exclusivos é controversa e o seu papel no controle e alívio dos sintomas não são bem descritos ${ }^{8,9}$.

Desse modo, o monitoramento da qualidade de terapias nutricionais nesse grupo de indivíduos é essencial para que - paciente tenha acesso ao melhor que a terapia possa Ihe oferecer, minimizando riscos e otimizando os recursos humanos e materiais empregados nessa terapêutica ${ }^{10}$. $O$ objetivo do estudo foi aplicar os indicadores de qualidade da TNE em pacientes com câncer em cuidados paliativos exclusivos.

\section{MÉTODO}

Trata-se de um estudo descritivo, observacional, de abordagem quantitativa. Elegeu-se como campo de aplicação da pesquisa o Hospital do Câncer IV, unidade de cuidados paliativos do Instituto Nacional de Câncer José Alencar Gomes da Silva, localizada na cidade do Rio de Janeiro - Brasil.

Os pacientes foram acompanhados durante a internação hospitalar, sendo incluídos aqueles com idade maior ou igual a 18 anos, de ambos os sexos, internados no período de maio a novembro de 2019, com cateter nasoentérico, nasogástrico, gastrostomia ou jejunostomia para infusão de dieta enteral, diagnosticados com câncer em estágio avançado independentemente da localização, capazes de responder adequadamente as informações necessárias ou que estavam com acompanhante capaz de fornecê-las. Foram excluídos pacientes com Karnofsky Performance Status (KPS) ${ }^{11}$ inferior a $30 \%$ no momento da avaliação inicial e que estavam recebendo alimentação via oral associada, para complementação do aporte energético.

Foram selecionados sete indicadores de qualidade da TNE, propostos pela ILSI-Brasil, escolhidos considerando sua aplicabilidade de acordo com características da unidade de atendimento. Os indicadores aplicados e as suas respectivas fórmulas são apresentados no Quadro 1.

Para os cálculos de adequação da administração calórica e proteica às necessidades nutricionais dos pacientes foram consideradas as recomendações da European Society of Parenteral and Enteral Nutrition (ESPEN) para pacientes com câncer, que estabelece 25 a $30 \mathrm{kcal} / \mathrm{kg}$ de peso e 1,0 a 1,5 $\mathrm{g}$ de proteína/ $\mathrm{kg}$ de peso ${ }^{12}$. Além disso, foram quantificados os motivos da suspensão ou interrupção da dieta enteral durante a internação.

Todos os dados foram coletados em prontuários eletrônicos e em fichas de acompanhamento nutricional do serviço de nutrição da unidade. Os pacientes foram acompanhados desde a internação hospitalar ou início da TNE até a alta ou óbito.

\section{Análise Estatística}

Os dados foram analisados pelo programa IBM SPSS Statistics versão 20. A análise descritiva foi apresentada em percentuais, para as variáveis categóricas, e em média e desvio padrão, para as variáveis contínuas. Os indicadores de qualidade foram descritos em percentuais.

\section{Aspectos Éticos}

O projeto foi aprovado pelo Comitê de Ética em Pesquisa do Instituto Nacional de Câncer (CEP/INCA), sob n ${ }^{\circ}$ CAAE 10586019.0.0000.5274.

Todos os indivíduos envolvidos nesta pesquisa assinaram o Termo de Consentimento Livre e Esclarecido (TCLE). 
Quadro 1 - Indicadores utilizados para avaliar a qualidade da terapia nutricional.

\begin{tabular}{|c|c|c|}
\hline Indicadores & Fórmula & Meta \\
\hline $\begin{array}{l}\text { Frequência de saída inadvertida de sonda de nu- } \\
\text { trição enteral }\end{array}$ & $\begin{array}{l}\mathrm{N}^{\circ} \text { de saída inadvertida da sonda enteral } \times 100 / \mathrm{N}^{\circ} \text { total de pacientes } \\
\text { em TNE } \times \mathrm{N}^{\circ} \text { de dias com sonda enteral }\end{array}$ & $<10 \%$ \\
\hline $\begin{array}{l}\text { Frequência de dias de administração adequada do } \\
\text { volume prescrito versus volume infundido* }\end{array}$ & $\begin{array}{l}N^{\circ} \text { de dias com volume adequado de } \operatorname{TNE}(\geq 80 \%) \times 100 / \\
N^{\circ} \text { total de dias em TNE }\end{array}$ & $\geq 80 \%$ \\
\hline $\begin{array}{l}\text { Frequência de dias de administração inadequada } \\
\text { de proteína* }\end{array}$ & $\begin{array}{l}N^{\circ} \text { de pacientes com aporte proteico inadequado } \times 100 / N^{\circ} \text { total de } \\
\text { paciente em TNE }\end{array}$ & $\leq 10 \%$ \\
\hline $\begin{array}{l}\text { Frequência de dias de administração inadequada } \\
\text { de energia }\end{array}$ & $\begin{array}{l}\mathrm{N}^{\circ} \text { de dias com aporte calórico menor que } 25 \% \text { de } 25 \mathrm{kcal} / \mathrm{kg} \text { ou } \\
\text { maior que } 25 \% \text { de } 30 \mathrm{kcal} / \mathrm{kg} \times 100 / \mathrm{N}^{\circ} \text { de dias em TNE }\end{array}$ & $<20 \%$ \\
\hline Frequência de diarreia & $\begin{array}{l}N^{\circ} \text { de pacientes em TNE que apresentam diarreia (três ou mais } \\
\text { evacuações líquidas por dia) x 100/ } N^{\circ} \text { total de pacientes em TNE }\end{array}$ & $\leq 10 \%$ \\
\hline Frequência de episódios de distensão abdominal & $\begin{array}{l}N^{\circ} \text { de pacientes em TNE que apresentam distensão abdominal x 100/ } \\
N^{\circ} \text { total de pacientes em TNE }\end{array}$ & $<15 \%$ \\
\hline Frequência de episódios de constipação intestinal & $\begin{array}{l}N^{\circ} \text { de pacientes em TNE que apresentam obstipação (menos } 3 \text { eva- } \\
\text { cuações por semana) x 100/ } N^{\circ} \text { total de pacientes em TNE }\end{array}$ & $<20 \%$ \\
\hline
\end{tabular}

Fonte: International Life Sciences Institute-Brasili ${ }^{14}$. TNE: Terapia Nutricional Enteral. *Fórmula adaptada

\section{RESULTADOS}

A amostra foi composta por 51 pacientes, com idade média de 60,8 \pm 12,3 anos, sendo $66,7 \%$ do sexo masculino e $33,3 \%$ do sexo feminino. A média de volume de dieta infundido no período estudado foi de $795 \pm 303 \mathrm{ml} / \mathrm{dia}$, o tempo total em TNE foi de 488 dias, com média de 9,6 7,7 dias. Demais características estão presentes na Tabela 1 . câncer de cabeça e pescoço foi o mais prevalente $(70,6 \%)$. $\bigcirc$ KPS inicial de 30 a 40 foi o mais comum no início da TNE (64,7\%), a disfagia total foi a indicação da TNE mais observada na amostra $(64,7 \%)$ e o cateter nasoentérico foi a via de administração mais utilizada $(54,9 \%)$.

A Tabela 2 apresenta os indicadores de qualidade utilizados, os resultados baseados nas fórmulas descritas e suas respectivas metas. Observa-se que as frequências de saída inadvertida de sonda de nutrição enteral, dias de administração adequada do volume prescrito versus volume infundido e diarreia estiveram dentro da meta estabelecida. Houve inadequação na administração de proteína e energia, assim como nas frequências dos episódios de distensão abdominal e constipação intestinal.

Durante a internação, ocorreram 71 episódios de interrupção ou suspensão da dieta enteral, e os motivos desses episódios são apresentados na Figura 1. A ocorrência de vômitos e os cuidados ao fim de vida (risco de óbito iminente) foram os mais comuns, 15,5\% e 14,1\%, respectivamente, seguidos de obstrução de sonda (12,7\%), sangramento volumoso $(8,5 \%)$, jejum para exames ou outros procedimentos $(8,4 \%)$, plenitude $(7 \%)$ e náuseas $(7 \%)$.
Tabela 1 - Características dos pacientes em terapia nutricional enteral.

\begin{tabular}{lc}
\hline Variáveis & $\mathbf{n}(\%)$ \\
\hline Localização do tumor primário & \\
Cabeça e pescoço & $36(70,6)$ \\
Trato gastrointestinal & $9(17,7)$ \\
Mama & $2(3,9)$ \\
Pulmão & $2(3,9)$ \\
Outros & $2(3,9)$ \\
\hline KPS inicial & \\
30 a 40\% & $33(64,7)$ \\
$\geq 50 \%$ & $18(35,3)$ \\
\hline
\end{tabular}

\section{Indicação da TNE}

Disfagia total

$33(64,7)$

Disfagia parcial

$10(19,6)$

Obstrução do TGI alto

Outros

$3(5,9)$

\section{Via de administração}

Cateter nasoentérico

$28(54,9)$

Gastrostomia

$22(43,1)$

Jejunostomia

$1(2,0)$

KPS: Karnofsky Performance Status; TNE: Terapia Nutricional Enteral. 


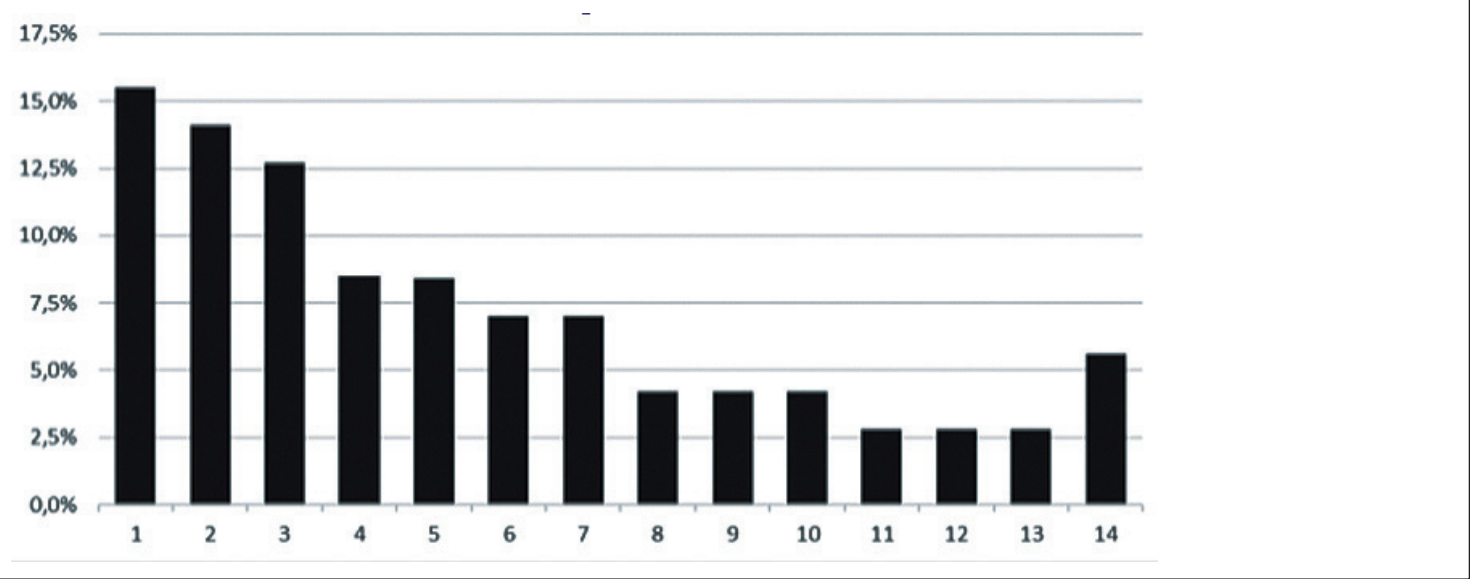

Figura 1 - Fatores determinantes para a interrupção ou suspensão da dieta enteral. 1: Vômitos; 2: Cuidados ao fim de vida; 3: Obstrução de sonda; 4: Sangramento volumoso; 5: Jejum para exames ou outros procedimentos; 6: Plenitude gástrica; 7: Náuseas; 8: Distensão abdominal; 9: Óbito; 10: Recusa do paciente; 11: Broncoaspiração; 12: Saída inadvertida de sonda; 13: Extravasamento de dieta; 14: Outros.

Tabela 2 - Frequências dos indicadores de qualidade da terapia nutricional enteral em pacientes oncológicos em cuidados paliativos exclusivos e suas respectivas metas.

\begin{tabular}{lcc}
\hline Indicadores & Resultado & Meta \\
\hline $\begin{array}{l}\text { Frequência de saída inadvertida de } \\
\text { sonda de nutrição enteral }\end{array}$ & $0,02 \%$ & $<10 \%$ \\
$\begin{array}{l}\text { Frequência de dias de administração } \\
\text { adequada do volume prescrito versus } \\
\text { volume infundido }\end{array}$ & $92,6 \%$ & $\geq 80 \%$ \\
$\begin{array}{l}\text { Frequência de dias de administração } \\
\text { inadequada de proteína }\end{array}$ & $25,5 \%$ & $\leq 10 \%$ \\
$\begin{array}{l}\text { Frequência de dias de administração } \\
\text { inadequada de energia }\end{array}$ & $57,4 \%$ & $<20 \%$ \\
$\begin{array}{l}\text { Frequência de diarreia } \\
\text { Frequência de episódios de distensão }\end{array}$ & $33,3 \%$ & $<10 \%$ \\
abdominal & $2,0 \%$ & \\
$\begin{array}{l}\text { Frequência de episódios de } \\
\text { constipação intestinal }\end{array}$ & $86,3 \%$ & $<20 \%$ \\
\hline
\end{tabular}

\section{DISCUSSÃO}

No presente estudo, foi observado que a maioria dos pacientes possuia câncer de cabeça e pescoço. Este, devido sua localização anatômica, pode comprometer significativamente a ingestão de alimentos, como consequência do comprometimento dos mecanismos de deglutição pelo próprio tumor, ou por complicações após cirurgia, radioterapia ou quimioterapia ${ }^{13}$. Por isso, a utilização de uma via alimentar acessória torna-se necessária, o que se justifica pela alta taxa de indicação de TNE por disfagia total nesse estudo.
Em relação aos indicadores, o relacionado à complicação mecânica da sonda de nutrição enteral, a frequência de saída inadvertida de sonda, esteve adequado no presente estudo $(0,02 \%)$. Um outro estudo que incluiu pacientes com doenças do trato gastrointestinal e cardiovasculares também encontrou adequação nesse indicador, com 1,2\% de frequência ${ }^{14}$. Já em um hospital universitário com 120 pacientes, em clínica médica e Unidade de Terapia Intensiva (UTI), que incluiu pacientes com doenças pulmonares, sepse e acidente vascular cerebral, foi encontrada inadequação da saída inadvertida de sonda $(31 \%)^{15}$. A avaliação desse indicador é importante para um melhor controle de complicações da sondagem, desperdício de dietas enterais e oferta nutricional diminuída nos pacientes'.

A frequência de dias de administração adequada do volume prescrito versus volume infundido também esteve dentro da meta estabelecida no presente estudo, ou seja, o desperdício de dietas foi mínimo, e os pacientes em geral receberam mais que $80 \%$ do volume prescrito. Já um estudo com pacientes em cuidados intensivos obteve $77,7 \%$ de volume infundido em relação ao prescrito, abaixo da meta estabelecida, neste caso, a administração inadequada teve correlação com o uso de drogas vasoativas e jejum maior que 24 horas $^{16}$. Outro estudo também encontrou inadequação do volume infundido em 144 pacientes com diversos tipos de doenças, com exclusão dos cuidados paliativos, que estavam internados em enfermarias clínicas. Mesmo considerando como adequada a administração de $70 \%$ da dieta, o valor encontrado foi de $65,3 \%$. Os autores justificam esse resultado com a ocorrência dos jejuns prolongados pré e pós-operatórios e realização de exames ${ }^{14}$. Esses procedimentos são menos comuns nos cuidados paliativos exclusivos. 
Os indicadores relacionados ao controle clínico avaliados foram: frequência de diarreia, constipação e distensão abdominal. A frequência de diarreia ficou dentro da meta proposta na literatura, apenas $2 \%$ dos pacientes apresentaram, enquanto a frequência de constipação intestinal e distensão abdominal foram inadequados, $86,3 \%$ e 33,3\%, respectivamente. Da mesma forma, Lee et al. ${ }^{3}$ encontraram adequação da frequência de diarreia $(8,1 \%)$ e inadequação da frequência de constipação $(28,6 \%)$ em estudo com pacientes com câncer em tratamentos antineoplásicos. Outro estudo com pacientes oncológicos também encontrou um percentual acima do preconizado de constipação intestinal (29\%) e distensão abdominal $(22,6 \%)^{17}$.

A constipação é um distúrbio muito comum em pacientes com câncer com doença avançada, devido a fatores como: medicamentos analgésicos, principalmente opioides; restrição ao leito; compressão do tumor sobre o intestino; danos neurológicos, que alteram a motilidade intestinal e falta de privacidade, podendo causar aumento abdominal, dor, náuseas e vômitos. O tratamento tanto dietético quanto farmacológico da constipação é fundamental, para assegurar a qualidade de vida do paciente e o controle satisfatório da dor 9 ,18.

A administração inadequada de energia $(57,4 \%)$ e proteína $(25,5 \%)$ também foi acima da meta estabelecida. Resultado semelhante foi encontrado em um estudo com pacientes em UTI, com inadequação calórica de $56,67 \%$ e proteica de $66,67 \%{ }^{19}$ e no estudo de Alves et al. ${ }^{20}$, onde a inadequação calórico-proteica foi de 55,03\%, em pacientes internados em enfermaria clínica médica.

É importante reconhecer que os objetivos da terapia nutricional em cuidados paliativos mudam conforme a doença avança, devendo sempre ser baseado no prognóstico do paciente $^{21}$. Segundo a ESPEN ${ }^{12}$, se a sobrevida esperada for de vários meses ou anos, a terapia nutricional deve ser administrada com o objetivo de garantir uma ingestão adequada de energia e proteína, manter um status de desempenho adequado e qualidade de vida. No entanto, em pacientes com menor sobrevida, a terapia nutricional pode ser usada com o objetivo de fornecer benefício principalmente sintomático, baseado no conforto, e não mais na adequação nutricional.

A perda acelerada de tecido muscular e adiposo que ocorre em pacientes com câncer avançado impacta na capacidade funcional, autonomia do paciente e qualidade de vida. Por isso, a identificação precoce dos pacientes que podem se beneficiar da terapia nutricional é necessária, com o objetivo de retardar ou evitar a desnutrição e caquexia nesses pacientes ${ }^{22}$.

Quanto aos fatores determinantes para a interrupção ou suspensão da dieta enteral durante a internação, os episódios de vômitos foram os mais observados, seguidos pelos cuidados ao fim de vida, obstrução de sonda, sangramento volumoso, jejum para exames ou outros procedimentos, plenitude gástrica e náuseas. Alves et al. ${ }^{17}$ também identificaram intercorrências semelhantes em pacientes com câncer, como realização de exames e ocorrência de vômitos. Souza et al. ${ }^{23}$, em um hospital oncológico, também encontraram entre as principais intercorrências obstrução de sonda e náuseas. Apesar do indicador de administração versus infusão da dieta ter tido um resultado adequado, o controle dos eventos preveníveis que levam à interrupção da dieta enteral deve ser realizado, a fim de minimizar divergências na administração da dieta enteral e possibilitar aporte nutricional adequado ao paciente.

Dentre as limitações do estudo pode-se destacar o número reduzido de participantes, um curto período de avaliação, heterogeneidade da amostra, e coleta de dados secundários. Entretanto, o presente estudo foi o primeiro a aplicar os indicadores de qualidade em cuidados paliativos exclusivos, podendo apresentar o cenário da assistência nutricional nesses pacientes e a identificação de aspectos a serem remodelados.

\section{CONCLUSÃO}

O uso dos indicadores de qualidade da terapia nutricional se mostrou viável para pacientes com câncer avançado em cuidados paliativos. $\bigcirc$ monitoramento dos resultados é essencial para identificação de possibilidades de melhorar a qualidade da terapia, visando à manutenção do estado nutricional e à capacidade de desempenho de funções desses pacientes, logo, repercutindo diretamente em sua qualidade de vida.

Algumas inadequações encontradas nesta população podem estar associadas à mudança de objetivo da terapia nutricional nos pacientes com menor expectativa de vida. Adaptações baseadas no prognóstico podem ser essenciais, além da aplicação de novos indicadores e adequações das metas propostas. Diante dos múltiplos fatores que podem influenciar nos objetivos da terapia nutricional nesses pacientes e a escassez de pesquisas na área, faz-se necessário o desenvolvimento de mais estudos.

\section{REFERÊNCIAS}

1. Waitzberg DL. Indicadores de qualidade em terapia nutricional: 10 anos de IQTN no Brasil: resultados, desafios e propostas. $3^{\mathrm{a}}$ ed. São Paulo: ILSI Brasil; 2018.

2. Oliveira Filho RS, Ribeiro LM, Caruso L, Lima PA, Damasceno NR, Soriano FG. Quality indicators for enteral and parenteral nutrition therapy: application in critically ill patients "at nutritional risk". Nutr Hosp. 2016;33(5):563.

3. Lee A, Oliveira Filho RS, Cardenas TC, Ozório GA, Gropp JPL, Waitzberg DL. Quality control of enteral nutrition therapy in cancer patients at nutritional risk. Nutr Hosp. 2017;34(2):264-70. 
4. World Health Organization. Definition of palliative care [internet]. 2017. [citado 2019 maio 20]. Disponível em: https:// www.who.int/cancer/palliative/definition/en/

5. World Health Organization. Cancer control: palliative care [internet]. 2007. [citado 2019 maio 20]. Disponível em: https:// www.who.int/cancer/publications/cancer_control_palliative/ en/

6. Benarroz MO, Faillace GBD, Barbosa LA. Bioética e nutrição em cuidados paliativos oncológicos em adultos. Cad Saúde Pública. 2009;25(9):1875-82.

7. Fearon K, Strasser F, Anker SD, Bosaeus I, Bruera E, Fainsinger $\mathrm{RL}$, et al. Definition and classification of cancer cachexia: an international consensus. Lancet Oncol. 2011;12(5):489-95.

8. Maillet JO, Potter RL, Heller L. Position of the American Dietetic Association: ethical and legal issues in nutrition, hydration, and feeding. J Am Diet Assoc. 2002;102(5):716-26.

9. Corrêa PH, Shibuya E. Administração da terapia nutricional em cuidados paliativos. Rev Bras Cancerol. 2007;53(3):317-23.

10. Waitzberg DL, Enck CR, Miyahira NS, Mourão JRP, Faim MMR, Oliseski M, et al. Terapia nutricional: indicadores de qualidade [internet]. 2011. [citado 2019 jul. 07]. Disponível em: https://diretrizes.amb.org.br/_BibliotecaAntiga/terapia_nutricional_indicadores_de_qualidade.pdf.

11. Mor $\bar{V}$, Laliberte $\bar{L}$, Morris JN, Wiemann M. The Karnofsky Performance Status Scale: an examination of its reliability and validity in a research setting. Cancer. 1984;53(9):2002-7.

12. Arends J, Bachmann P, Baracos V, Barthelemy N, Bertz H, Bozzetti F, et al. ESPEN guidelines on nutrition in cancer patients. Clin Nutr. 2017;36(1):11-48.

13. Raykher A, Russo L, Schattner M, Schwartz L, Scott B, Shike M. Enteral nutrition support of head and neck cancer patients. Nutr Clin Pract. 2007;22(1):68-73.
14. Machado SKC, Alves TCHS. Aplicação de indicadores de qualidade em terapia nutricional enteral em um hospital público de Salvador-BA. BRASPEN J. 2018;33(3):320-5.

15. Oliveira JS, Oliveira CC. Quality indicators in enteral nutrition therapy at a university hospital. BRASPEN J. 2019;34(2):180-6.

16. Arcoverde GMPF, Pinzon CDM, Pereira CGS, Melo NCO, Alves DVS, Medeiros RAB, et al. Adequação nutricional de pacientes em unidade de terapia intensiva segundo indicadores de qualidade em terapia nutricional. Nutr Clin Diet Hosp. 2019;39(1):83-92.

17. Alves TP, Barbosa JM, Veras LN, Cabral NSG. Avaliação da qualidade da terapia nutricional enteral ofertada ao paciente oncológico hospitalizado. BRASPEN J. 2019;34(3):239-44.

18. Agra G, Fernandes MA, Platel ICS, Barros NCB, Freire MEM. Constipação em pacientes com doença oncológica avançada em uso de opioides. Mundo saúde. 2013;37(4):472-8.

19. Barroso ACS, Cavalcante AS, Marques SSF, Sató ALSA. Comparação entre necessidade, prescrição e infusão de dietas enterais em um hospital público de Belém-PA. BRASPEN J. 2019;34(1):46-51.

20. Alves AHR, Borges S. Indicadores de qualidade em terapia enteral: avaliação da assistência nutricional ao paciente hospitalizado. BRASPEN J. 2019;34(1):77-82.

21. Hui D, Dev R, Bruera E. The last days of life: symptom burden and impact on nutrition and hydration in cancer patients. Curr Opin Support Palliat Care. 2015;9(4):346-54.

22. Tan CS, Read JA, Phan VH, Beale PJ, Peat JK, Clarke SJ. The relationship between nutritional status, inflammatory markers and survival in patients with advanced cancer: a prospective cohort study. Support Care Cancer. 2015;23(2):385-91.

23. Souza IA, Bortoletto MM, Dias AMN, Almeida NM, Ribeiro LC, Mendonça EG. Nutrição enteral em pacientes oncológicos: diferenças entre o que é prescrito e administrado. Nutr Clín Diet Hosp. 2018;38(2):31-8.

Local de realização do estudo: Instituto Nacional de Câncer José Alencar Gomes da Silva (INCA), Rio de Janeiro, RJ, Brasil.

Conflito de interesse: Os autores declaram não haver. 